ESAIM: PROCEEDINGS, April 2012, Vol. 36, p. 106-120

Danièle Fournier-Prunaret, Laura Gardini, \& Ludwig Reich, Editors

\title{
ORGANIZING CENTERS \\ IN PARAMETER SPACE OF DISCONTINUOUS 1D MAPS. THE CASE OF INCREASING/DECREASING BRANCHES
}

\author{
Laura Gardini $^{1}$, Viktor Avrutin ${ }^{2}$, Michael Schanz $^{2}$, Albert Granados ${ }^{2}$ and \\ IRYNA SUSHKO ${ }^{3}$
}

\begin{abstract}
This work contributes to classify the dynamic behaviors of piecewise smooth systems in which border collision bifurcations characterize the qualitative changes in the dynamics. A central point of our investigation is the intersection of two border collision bifurcation curves in a parameter plane. This problem is also associated with the continuity breaking in a fixed point of a piecewise smooth map. We will relax the hypothesis needed in [4] where it was proved that in the case of an increasing/decreasing contracting functions on the left/right side of a border point, at such a crossing point, we have a big-bang bifurcation, from which infinitely many border collision bifurcation curves are issuing.
\end{abstract}

AMS (2000) subject classification. 37E05, 37G10, 37G35.

Keywords. piecewise smooth maps, border collision bifurcations, organizing centers.

Résumé. Cet travail est une contribution à la classification des comportements dynamiques de systèmes réguliers par morceaux dans lesquels les bifurcations de collision au bord caractérisent les changements qualitatifs de la dynamique. Un point central de notre étude est l'intersection de deux courbes de bifurcation de colision au bord dans un plan de paramètre. Ce problème est aussi associé avec la rupture de continuité en un point fixe d'une application régulière par morceaux. Nous allons relacher l'hypothèse requise dans [4], où il a été montré que dans le cas de fonctions contractantes croissantes/décroissantes strictement à gauche/droite d'un point du bord, en un tel point de franchissement, nous avons une bifurcation big-bang, de laquelle est issue une infinité de courbes de bifurcation de collision au bord.

Mots clefs. applications régulières par morceaux, bifurcations de collision au bord, centres organisateurs.

\section{INTRODUCTION}

The problem of a classification of the phenomena which may occur at a border collision bifurcation ${ }^{1}$ (BCB for short) in a piecewise smooth one-dimensional discontinuous map is a problem started several years ago, and it is far from being completely understood. However, the first results are due to Leonov ( $[11,12]$, see

\footnotetext{
${ }^{1}$ DESP, University of Urbino, Urbino, Italy laura.gardini@uniurb.it.

${ }^{2}$ Institute of Parallel and Distributed Systems, University of Stuttgart, Germany; emails: Viktor .Avrutin@ipvs .uni-stuttgart.de, Michael.Schanz@ipvs.uni-stuttgart.de, Albert Granados@ipvs.uni-stuttgart.de.

${ }^{3}$ National Academy of Sciences of Ukraine, and Kiev School of Economics, Kiev, Ukraine; email: sushko@imath.kiev.ua.

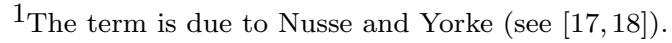

(C) EDP Sciences, SMAI 2012 
also in [13], where several cases are classified), and since then several authors have published relevant works, which contribute to the progress in the comprehension of the bifurcation mechanisms and related phenomena. We recall [1-3,6,8], where applied models were investigated, and [5,10], where the map in canonical form is considered, and the Leonov's approach improved. Further results are shown in [22], where assuming a piecewise linear map with three partitions, it is proved how the so-called period adding scheme can be used. These bifurcation mechanisms are quite important for their role in applied models, in several contexts. In engineering, see $[9,14-16,23]$, but also in economics (see [7, 19-21]).

As already noticed in several works, the BCB curves form complex structures in 2D parameter planes, typically governed by some organizing centers (codimension-2 bifurcations). Especially interesting in this context are the so-called big bang bifurcations (BBB for short), defined by the condition that an infinite number of BCB curves are issuing from the bifurcation point ( $[1])$. Considering a piecewise-smooth one-dimensional map, when it has two periodic orbits undergoing BCBs at the curves $\Phi_{L}$ and $\Phi_{R}$ which are intersecting at some point $\mathrm{P}$, then in many cases it was observed by numerical experiments that the point $\mathrm{P}$ represents a BBB point. The questions that we have to consider are: (a) Under which conditions the intersection point of two BCB curves represents a $\mathrm{BBB}$ point? (b) If the intersection point of two $\mathrm{BCB}$ curves represents a $\mathrm{BBB}$ point, what are the orbits whose existence regions are bounded by the infinitely many BCB curves issuing from the BBB point? As we shall see below, this dynamic problem is also associated with the continuity breaking in a fixed point of a piecewise smooth map, and all the possible cases can be classified depending on the shape of the two functions involved on the right/left sides of the fixed point. The partial results are given in [4], where it is shown that assuming two contracting functions, one increasing and one decreasing on the two sides of the fixed point, the BBB occurs, leading to stable cycles of any period, with periodicity regions which are overlapping in pair.

The object of the present work is to characterize the case under the generic assumption of one increasing and one decreasing function on the two sides of the fixed point, showing that the BBB occurs. The existing cycles may be stable or unstable.

As recalled above, a crucial point is the intersection of two BCB curves. A BCB curve is the locus of points in the parameter space at which a periodic point of an existing cycle is colliding with the break point, which here, without loss of generality, is set to $x=0$. Each locus of a BCB separate two regions, on one side the cycle exists, while on the other side the cycle does not exist. This is independent of the stability of the cycle, which, as usual, comes from the modulus of its eigenvalue, given by the products of the derivatives in the periodic points. Let us consider the intersection of two BCB curves, $\Phi_{L}$ and $\Phi_{R}$, boundaries of two different periodicity regions, $\Pi\left(p_{l}\right)$ and $\Pi\left(p_{r}\right)$ of a given piecewise smooth map $F$. $\Phi_{L}$ is the BCB curve such that when a parameter point belongs to it the map $F$ has a periodic point $x_{l}^{*}$ (of prime period $p_{l}$ and associated with a symbolic sequence $\left.\rho=\rho_{1} \ldots \rho_{p_{l}}\right)$ colliding with the point $x=0$ from the left side $L$, while on the other BCB curve, $\Phi_{R}$, the periodic point $x_{r}^{*}$ (of prime period $p_{r}$ and associated with a symbolic sequence $\sigma=\sigma_{1} \ldots \sigma_{p_{r}}$ ) of $F$ is colliding with $x=0$ from the right side $R$. Let us define as $H_{l}(x)$ (resp. $H_{r}(x)$ ) the composite function which gives the periodic point colliding with $x=0$ from the left (resp. right) side. That is: $H_{l}(x)=F_{\rho_{p_{l}}} \circ \ldots F_{\rho_{1}}(x)$ and $H_{r}(x)=F_{\sigma_{p_{r}}} \circ \ldots F_{\sigma_{1}}(x)$ so that $H_{l}\left(x_{l}^{*}\right)=x_{l}^{*}$ and $H_{r}\left(x_{r}^{*}\right)=x_{r}^{*}$.

As we are considering BCBs involving the unique discontinuity point $(x=0)$, when a parameter point belongs to the overlapping region then the map $F$ possesses both cycles, while in the non overlapped regions only one of them exists. It follows that the crossing of two BCB curves in a point $P$ implies the existence of the four regions shown qualitatively in Fig. 1. When the dynamics of $F$ are bounded into an absorbing interval, say $I$, which does not include stable fixed points, then some other attracting set must exist.

Notice that it is not necessary to require a specific number of components of the map $F$, also it is not necessary to require that the periods $\left(p_{l}\right.$ and $\left.p_{r}\right)$ are different. In any case, the reasoning at the border collision bifurcations which involve one discontinuity point $x=0$ may be done as here described, considering the map $T$ defined via the two functions $T_{L}$ and $T_{R}$ as follows:

$$
x^{\prime}=T(x), \quad T(x)= \begin{cases}T_{L}(x)=\mu_{l}+H_{l}(x) & \text { if } \quad x<0 \\ T_{R}(x)=\mu_{r}+H_{r}(x) & \text { if } \quad x>0\end{cases}
$$




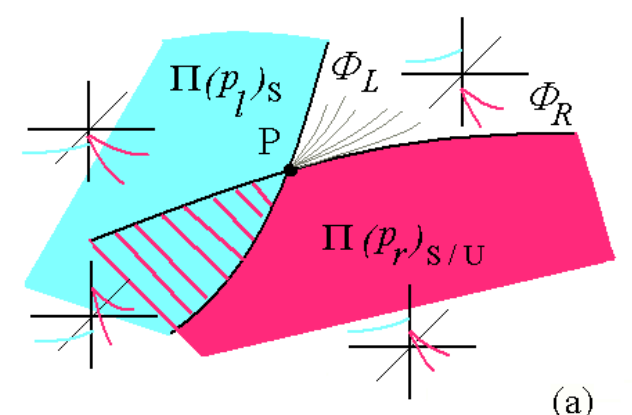

(a)

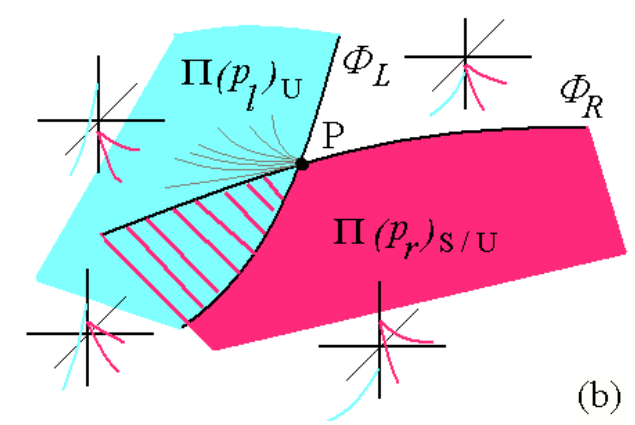

(b)

FiguRE 1. Intersection of two periodicity regions in a BBB point $P$. The cycle $p_{l}$ is stable in (a) and unstable in (b). The cycle $p_{r}$ may be stable or unstable.

where we know that when the parameters belong to the point $P$ (in $\Phi_{L} \cap \Phi_{R}$ ) then we necessarily have $\mu_{l}=\mu_{r}=0$. When a parameter point belongs to $\Phi_{L}$ then $T_{L}(0)=0$ occurs, that is $\mu_{l}=0$ and the eigenvalue of the cycle with periodic point $x_{l}^{*}=0$ is given by $\lambda_{l}=H_{l}^{\prime}(0)$. Similarly, when a parameter point belongs to $\Phi_{R}$ then $T_{R}(0)=0$ occurs, that is $\mu_{R}=0$ and the eigenvalue of the cycle with periodic point $x_{r}^{*}=0$ is given by $\lambda_{r}=H_{r}^{\prime}(0)$.

Here we are investigating the case of increasing/decreasing branches on the two sides of $x=0$, which is topologically conjugated with the case of decreasing/increasing branches, as it is easy to see. The particular case in which, in addition, the colliding fixed points are assumed to be stable just before the bifurcation (that is, both $H_{l}$ and $H_{r}$ are contractive functions near $x=0$ ) was studied in [4], where it was shown that the BBB occurs leading to the appearance of stable cycles of any period with periodicity regions overlapping in pair. In the present work, however, we give a different proof and extend the results presented there permitting the colliding fixed points to be unstable. In particular, we show in Theorem 3 that the same situation holds near the BBB point when, under some restrictions, only $x_{r}^{*}$ (the fixed point with negative eigenvalue) is unstable.

The plane of the work is as follows. In the next section we reformulate and generalize some of the arguments presented in [4] in terms of an appropriate first return map. In Sec. 3 we present our results and their proofs, illustrated by several examples. Additionally, in Sec. 4 we discuss the possible situations in the case that after the bifurcation the fixed point with the positive eigenvalue is virtual and the one with the negative eigenvalue exists.

\section{First RETURN MAP}

We can generalize the problem presented in the introduction to that of a continuity breaking of some piecewise smooth function $f$, defined as follows:

$$
x^{\prime}=f(x), \quad f(x)=\left\{\begin{array}{lll}
f_{L}(x)=\mu_{l}+H_{l}(x) & \text { if } & x<0 \\
f_{R}(x)=\mu_{r}+H_{r}(x) & \text { if } & x>0
\end{array}\right.
$$

In this map, when the offset parameters are $\mu_{l}=\mu_{r}=0$ then the point $x=0$ is a fixed point: $H_{l}(0)=0$ and $H_{r}(0)=0$. According to the task of this paper we assume that the function $H_{l}$ is increasing in a left neighborhood of $x=0$ and the function $H_{r}$ is decreasing in a right neighborhood of $x=0$.

We also remark that we limit our analysis only to this case, as the "symmetric one", that means that the function $H_{l}$ is decreasing in a left neighborhood of $x=0$ and the function $H_{r}$ is increasing in a right neighborhood of $x=0$, is topologically conjugated to the first one. To obtain all the results for this second case it is enough to exchange the letters "left" and "right" in the symbolic sequences that we shell describe below.

Then, breaking the continuity (existing for $\mu_{l}=\mu_{r}=0$ ) we are interested in the dynamic behavior of $f$ when the offsets are varied in the parameter plane $\left(\mu_{r}, \mu_{l}\right)$ close to $(0,0)$. A qualitative picture is shown in Fig. 2. 


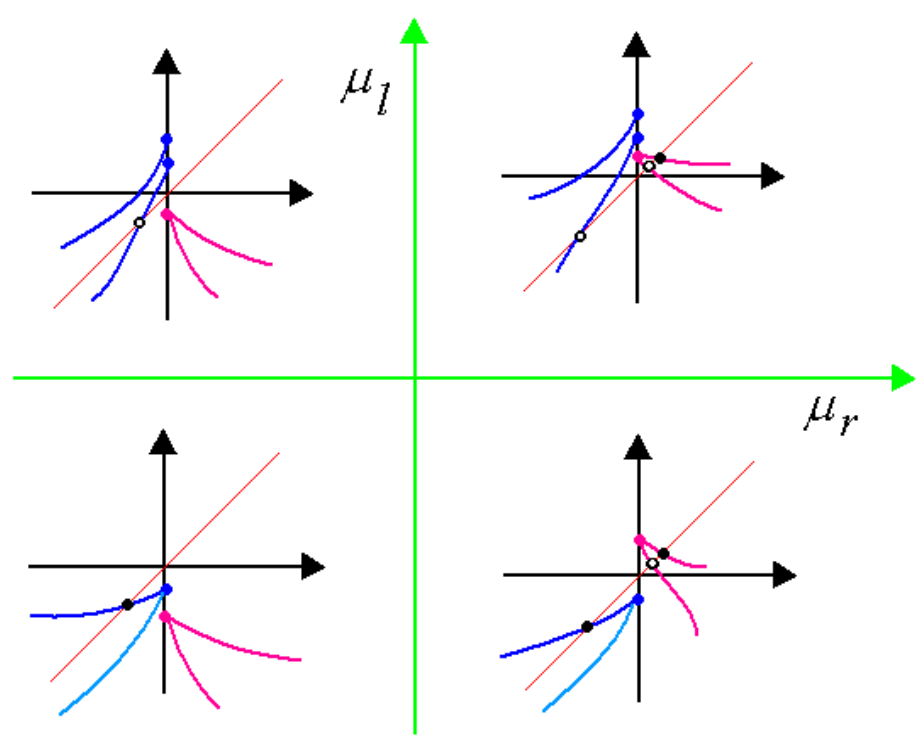

FiguRE 2. Different shapes for function $f$ for different combinations of the signs of $\mu_{l}$ and $\mu_{r}$. The contracting/expanding cases are shown. Black/white circles denote stable/unstable fixed points.

We notice that the region of existence of the fixed point $x_{l}^{*}$ on the left side depends on the slope of $H_{l}$ at the point colliding on the left side. Especially, if $H_{l}^{\prime}(0)<1$ the fixed point $x_{l}^{*}$ exists for $\mu_{l}<0$ and it is stable for $\mu_{l}$ in a left neighborhood of 0 . While for $H_{l}^{\prime}(0)>1$ the fixed point $x_{l}^{*}$ exists for $\mu_{l}>0$ and it is unstable. Differently, independently on the modulus of $H_{r}^{\prime}(0)<0$, the fixed point $x_{r}^{*}$ on the right side exists for $\mu_{r}>0$ and does not exist for $\mu_{r}<0$.

It is clear that one of the interesting regions in which we are led to study the dynamic behaviors is the upper left quadrant in Fig. 2, that is, $\mu_{r} \leq 0$ and $\mu_{l}>0$. In this sections we will consider this case. In this quadrant, the shape of the map is such that when we move the offsets from $(0,0)$ an absorbing interval $I$ exists, given by

$$
I=\left[f_{R} \circ f_{L}(0), f_{L}(0)\right]=\left[f_{R}\left(\mu_{l}\right), \mu_{l}\right]
$$

where $\mu_{l}>0$ and $f_{R}\left(\mu_{l}\right)<0$. Under our assumption, for $\mu_{r} \leq 0$ and $\mu_{l}>0$ close to $(0,0)$ the function $f_{L}(x)$ is increasing in $I$ for $x<0$, while $f_{R}(x)$ is decreasing in $I$ for $x>0$. As already mentioned, depending on the slope of $f_{L}(x)$ an unstable fixed point $x_{l}^{*}\left(\mu_{l}\right)$ may exist or not. When an unstable fixed point $x_{l}^{*}\left(\mu_{l}\right)$ exists, then the absorbing interval $I$ exists as long as the inequality $f_{R} \circ f_{L}(0)>x_{l}^{*}\left(\mu_{l}\right)$ holds, that means for

$$
f_{R}\left(\mu_{l}\right)>x_{l}^{*}\left(\mu_{l}\right)
$$

The bifurcation occurring at $f_{R}\left(\mu_{l}\right)=x_{l}^{*}\left(\mu_{l}\right)$ is a contact bifurcation leading to the destruction of the absorbing interval $I$, and for $f_{R}\left(\mu_{l}\right)<x_{l}^{*}\left(\mu_{l}\right)$ the absorbing interval does not exist any more. We are restricting our analysis to the constraint in (4), as this condition is sufficient to give a feed back mechanism, leading to a well defined first return map. Then, fixed any parameter value $\mu_{l}>0$ close to 0 , we decrease $\mu_{r}$ starting from 0 up to $f_{R}\left(\mu_{l}\right)=x_{l}^{*}\left(\mu_{l}\right)$ if a fixed point exists (see Fig. 2). Although the global dynamics of the map also depend on the nonlinear functions $f_{L}$ and $f_{R}$, under the only assumption of increasing/decreasing shape of the functions $H_{l}$ and $H_{r}$, we shall see that all the cycles with the symbolic sequence $R L^{k}$ must exist, with BCB curves in the parameter plane $\left(\mu_{r}, \mu_{l}\right)$ issuing from $(0,0)$.

From the assumptions that the function $f_{L}(x)$ is increasing and $f_{R}(x)$ decreasing, considering $\mu_{l}>0$ close to 0 , and $\mu_{r} \leq 0$, it follows that any initial condition in the right neighborhood of $x=0$ is mapped by $f_{R}(x)$ in 
the left side, from which $f_{L}(x)$ is applied as long as we obtain again a point in the right side. Thus the dynamic behavior of the map $f$ can be completely described by the first return map in the right side, that is, in the interval $J \subset I$ given by

$$
J=\left[0, f_{L}(0)\right]=\left[0, \mu_{l}\right] .
$$

For each point $x \in J, x \neq 0$, its first return value $F_{R}(x)$ is given by $f_{L}^{k} \circ f_{R}(x)$ where $k \geq 1$ is the first integer such that $f_{L}^{k} \circ f_{R}(x) \in J$.

Notice that a cycle of the map $f$ associated with a fixed point of the first return map $F_{R}$ is necessarily of the so-called maximal type. That is, it has only one periodic point on the right side, followed by one or more points on the left side, thus of the symbolic sequence $R L^{k}$. The periodic point on the right side (say $\left.x_{0}\right)$ of such a cycle is obtained as a solution of the equation $f_{L}^{k} \circ f_{R}\left(x_{0}\right)=x_{0}$. A BCB of this cycle occurs when $x_{0}=0$. Therefore, the equation

$$
f_{L}^{k} \circ f_{R}(0)=0
$$

describes one BCB curve bounding the periodicity region $\Pi\left(R L^{k}\right)$. The other BCB of the same cycle occurs when the periodic point $x_{k}$ collides with the boundary $x=0$ from the left side, or equivalently if the point $x_{0}$ collides with the right boundary of the absorbing interval $I$ (i.e. $J$ ), that is, collides with the point $\mu_{l}$. Hence, the corresponding BCB curve is given by $f_{L}^{k} \circ f_{R}\left(\mu_{l}\right)=\mu_{l}$, or, equivalently:

$$
f_{L}^{k} \circ f_{R} \circ f_{L}(0)=f_{L}(0)
$$

that is,

$$
f_{L}^{k-1} \circ f_{R} \circ f_{L}(0)=0
$$

where $f_{L}^{0}$ is the identity function. An immediate result is that the BCB curves given by Eqs. (6) and (7) associated with the periodicity region $\Pi\left(R L^{k}\right)$ intersect at the origin $(0,0)$ in the parameter plane $\left(\mu_{r}, \mu_{l}\right)$, as the equations are satisfied by $\mu_{r}=0$ and $\mu_{l}=0$, and cannot exist in the half-plane $\mu_{l}<0$, thus they necessary belong to the half-plane $\mu_{l}>0$.

The first return map $F_{R}(x)$ is clearly piecewise smooth, and discontinuous in the points where its definition changes, i.e. when the integer $k$ changes in the function $f_{L}^{k} \circ f_{R}(x)$ giving the first return in the interval $J$. The discontinuity points of $F_{R}(x)$ are given by the preimages of the origin $x=0$ located in the interval $J$, which, in their turn, depend on the shape of $f_{L}(x)$. However, the functions $f_{L}(x)$ and hence also $f_{L}^{k}(x)$ are increasing, thus invertible, as well as $f_{R}(x)$ is decreasing and thus invertible. So let us define the point $d_{k}$ as the first preimage of the origin belonging to the positive side, given by

$$
d_{k}:=f_{R}^{-1} \circ f_{L}^{-k}(0)
$$

Then it is clear that the map $F_{R}(x)$ is defined as $F_{R}(x):=f_{L}^{k} \circ f_{R}(x)$ for $x \in\left[0, d_{k}\right]$ with $F_{R}(0)=f_{L}^{k} \circ f_{R}(0)=$ $f_{L}^{k}\left(\mu_{r}\right) \in J\left(\operatorname{as} f_{L}^{k}\left(\mu_{r}\right) \in\left(0, \mu_{l}\right]\right)$ and at the point $d_{k}$ we have $F_{R}\left(d_{k}\right)=f_{L}^{k} \circ f_{R}\left(d_{k}\right)=0$. For this first branch of the first return map we immediately have that the graph of the function $F_{R}(x)$ is decreasing and intersecting the diagonal only once, that is, a fixed point of $F_{R}$ must exist (as the function $G(x)=F_{R}(x)-x$ takes values of opposite signs at the extrema of the interval $\left[0, d_{k}\right]$, and thus must have a zero which corresponds to a fixed point of $F_{R}$ ). If $d_{k}>\mu_{l}$ this is the only branch of the first return map, otherwise, if $d_{k}<\mu_{l}$, then the function $F_{R}$ is given by $f_{L}^{k+1} \circ f_{R}(x)$ for $x \in\left[d_{k}, d_{k+1}\right]$ where $d_{k+1}=f_{R}^{-1} \circ f_{L}^{-(k+1)}(0)$. So, for example, if $d_{k+1}>\mu_{l}$ then the first return map is defined by only two pieces

$$
F_{R}(x):=\left\{\begin{array}{lll}
T_{0}(x):=f_{L}^{k} \circ f_{R}(x), & \text { if } \quad 0 \leq x<d_{k} \\
T_{1}(x):=f_{L}^{k+1} \circ f_{R}(x), & \text { if } \quad d_{k}<x \leq \mu_{l}
\end{array}\right.
$$

where $T_{0}(0) \in\left(0, \mu_{l}\right], T_{0}\left(d_{k}\right)=0$, and we also have also that $T_{1}\left(d_{k}\right)=f_{L}^{k+1} \circ f_{R}\left(d_{k}\right)=f_{L} \circ f_{L}^{k} \circ f_{R}\left(d_{k}\right)=$ $f_{L}(0)=\mu_{l}>d_{k}, T_{1}\left(\mu_{l}\right) \in\left(0, \mu_{l}\right]$. Thus also $T_{1}(x)$ must be decreasing and must have a fixed point in the interval $\left[d_{k}, \mu_{l}\right]$. Similarly, if $d_{k+1} \leq \mu_{l}$, then we have $T_{1}(x)$ defined for $x \in\left[d_{k}, d_{k+1}\right]$ and $T_{2}(x)=f_{L}^{k+2} \circ f_{R}(x)$ 
for $x>d_{k+1}$, so that the function $F_{R}$ becomes defined on three pieces, and so on. It is clear that depending on the shape of the function $f_{L}(x)$, we can have also several preimages of the origin inside the interval $J$, that is several discontinuity points $d_{j}$, and each branch of definition of the first return map must be decreasing and with a unique fixed point.

In order to demonstrate that the dynamics of the first return map as defined above reflects completely the dynamics of the map $f$, we prove the following

Proposition. Suppose the function $H_{l}$ increasing in a left neighborhood of $x=0$ and the function $H_{r}$ decreasing in a right neighborhood of $x=0$. Then for ${ }^{2} \mu_{l}>0$ and $\mu_{r} \leq 0$ the first return map $F_{R}$ shows all the local and global properties and bifurcations of $f$.

Proof. Let us first consider the map $f$ with fixed parameters. For a given function $f$ we can construct the first return map $F_{R}$ as described above, determining all the discontinuity points $d_{k}, d_{k+1}, \ldots$ belonging to $J$, and the functions defining the components of $F_{R}$, which are given by $T_{0}(x)=f_{L}^{k} \circ f_{R}(x), T_{1}(x)=f_{L}^{k+1} \circ f_{R}(x), \ldots$ whereby the number of branches of $F_{R}$ does not play any role. Then for any initial condition $z \in J$ the trajectory of this point under application of $f$ is given by the sequence

$$
z: f(z), f^{2}(z), f^{3}(z), \ldots
$$

We can also specify explicitly the application of $f_{R}$ and $f_{L}$. Proceeding in this way, it is enough to declare which function is applied to the last computed point. For example, for the trajectory of the point $z$ shown in Fig. 5 this leads to

$$
z:\left[f_{R}, f_{L}\right],\left[f_{R}, f_{L}\right],\left[f_{R}, f_{L},, f_{L}\right],\left[f_{R}, f_{L}, f_{L}\right],\left[f_{R}, f_{L}\right], \ldots
$$

where the parenthesis are added in such a way that whenever we apply $f_{R}$ we close the previous parenthesis and open the new one. Then we have

$$
z:\left[f_{L} \circ f_{R}\right],\left[f_{L} \circ f_{R}\right],\left[f_{L}^{2} \circ f_{R}\right],\left[f_{L}^{2} \circ f_{R}\right],\left[f_{L} \circ f_{R}\right], \ldots
$$

which can also be written as

$$
z:\left[T_{0}(z)\right],\left[T_{0}\right],\left[T_{1}\right],\left[T_{1}\right],\left[T_{0}\right], \ldots
$$

and this is exactly the trajectory of $z$ under application of the first return map $F_{R}$

$$
z: F_{R}(z), F_{R}^{2}(z), F_{R}^{3}(z), \ldots
$$

The backward reasoning is also valid, since in a trajectory of $F_{R}$ we can "expand" the definitions of the maps and in this way we obtain exactly the trajectory under $f$ (see the trajectory of $z$ with $f$ in Fig. 5a and with $F_{R}$ in Fig. 5b, the points of the trajectory in $J$ clearly are the same). Thus also the stability of cycles and homoclinic orbits can be studied equivalently via $f$ and $F_{R}$.

As the parameters of $f$ change, the shape of $F_{R}$ also changes, i.e. the number and position of the discontinuity points $d_{k}$, and this implies that all the global bifurcations of $f$ associated with the break point can be studied by using $F_{R}$. Which means that these are associated with the exit/entrance of a new branch in the definition of $F_{R}$ (which also correspond to the exist/entrance of a new discontinuity point in $J$ ).

The exit of a branch in the definition of $F_{R}$ occurs at the merging of a discontinuity point $d_{k}$ with 0 , and $d_{k}=0$ leads to $f_{R}^{-1} \circ f_{L}^{-k}(0)=0$, that is

$$
d_{k}=0: f_{L}^{k} \circ f_{R}(0)=0
$$

The entrance of a branch in the definition of $F_{R}$ occurs at the merging of a discontinuity point $d_{j}$ with $\mu_{l}$, and $d_{j}=\mu_{l}$ leads to $f_{R}^{-1} \circ f_{L}^{-j}(0)=\mu_{l}$, that is $f_{L}^{j} \circ f_{R}\left(\mu_{l}\right)=0$ which corresponds to

$$
d_{j}=\mu_{l}: f_{L}^{j} \circ f_{R} \circ f_{L}(0)=0
$$

\footnotetext{
${ }^{2}$ The same property clearly holds also for $\mu_{r}>0$ assuming $F_{R}(x):=f(x)$ for $x \in\left(0, d_{0}\right), d_{0}=f_{R}^{-1}(0)$.
} 
which are the two BCB curves already obtained above.

\section{Continuity BREAKing}

\subsection{General case}

Let us prove the existence of all the periodicity regions associated with the maximal cycles corresponding to symbolic sequences $R L^{k}$.

Theorem 1. Consider the map $f$ in (2) assuming $H_{l}(0)=0=H_{r}(0)$, the function $H_{l}(x)$ increasing in a left neighborhood of $x=0$ and the function $H_{r}(x)$ decreasing in a right neighborhood of $x=0$. Then infinitely many $B C B$ curves are issuing from the point $(0,0)$ in the parameter plane $\left(\mu_{r}, \mu_{l}\right)(i . e .(0,0)$ is a big bang bifurcation point).

Proof. Consider the function $f_{L}^{k}(x)$ for any $k \geq 1$. For $\mu_{l}=0$ we have $f_{L}^{k}(x)=H_{l}^{k}(x)$ increasing for $x<0$ and $f_{L}^{k}(0)=H_{l}^{k}(0)=0$, for any $k>0$. Thus for $\mu_{l}>0$ close enough to 0 we have $f_{L}^{-k}(0)<0$ also close to zero. Then set $\mu_{r}=f_{L}^{-k}(0)$ so that we have $f_{R}^{-1} \circ f_{L}^{-k}(0)=0$ which implies that the point $\left(\mu_{r}, \mu_{l}\right)$ belongs to the BCB curve of a cycle $R L^{k}$ for $f$ which, as shown in the previous section, is necessarily issuing from the point $(0,0)$ in the $\left(\mu_{r}, \mu_{l}\right)$ plane. Then, by continuity of the function $f_{L}^{k} \circ f_{R}(x)$ in a right neighborhood of $x=0$, for a suitable value $\mu_{r}=f_{L}^{-k}(0)+\varepsilon$ there exists a point $x_{0}>0$ such that $f_{L}^{k} \circ f_{R}\left(x_{0}\right)=x_{0}$, i.e. a periodic point of a $(k+1)$-cycle of $f$ with symbolic sequence $R L^{k}$.

Notice that in the assumptions of Theorem 1 we require monotone functions ${ }^{3}$, and in the case of smooth functions their derivative may be $H_{l}^{\prime}(0) \geq 0$ and $H_{l}^{\prime}(x) \geq 0$ in a left neighborhood of the origin, and $H_{r}^{\prime}(0) \leq 0$ and $H_{r}^{\prime}(x) \leq 0$ in a right neighborhood of the origin.

It is clear that the proof given above is only an existence proof. It shows the existence of the BCB curves already written in (6). Each one, for any $k>0$, gives one boundary of the periodicity region associated with the cycle with symbolic sequence $R L^{k}$, the other side (for the same cycle $R L^{k}$ ) being the curve given in (7), which also is a BCB curve which must issue from the point $\left(\mu_{r}, \mu_{l}\right)=(0,0)$ of the parameter plane. Then more properties can be investigated on the structure of the existing periodicity regions.

For example we have the following

Property: for any $\mu_{l}>0$ at $\mu_{r}=0$ a 2-cycle $R L$ exists, assuming $f_{L}(x)$ increasing and $f_{R}(x)$ decreasing in $I$.

In fact, as already remarked, in such a case the function $f_{L} \circ f_{R}(x)$ is decreasing in $J$ and must have a fixed point, which means a 2 -cycle for $f$.

Also, notice that the structure formed by the periodicity regions associated with the maximal cycles of symbolic sequence $R L^{k}$ is different in the following two situations:

(i) an unstable fixed point $x_{l}^{*}\left(\mu_{l}\right)$ does not exist (as for $H_{l}^{\prime}(0)<1$ ),

(ii) an unstable fixed point $x_{l}^{*}\left(\mu_{l}\right)$ exists.

In case (i) the functions $f_{L}^{k}(x)$ become more and more flat as $k$ is increased, which implies that the BCB curves have as limit set the $\mu_{r}$-axis (an example is shown in Fig. 4). While in case (ii) the BCB have as limit set the curve defined by the constraint in (4) (i.e. the curve of equation $f_{R}\left(\mu_{l}\right)=x_{l}^{*}\left(\mu_{l}\right)$, see Fig. 7 for an example).

Before considering some particular cases in the next subsection, let us here remark the connection between our results stated above on the continuity breaking with the intersection point $P$ of two border collision bifurcation curves in a parameter plane, as described in the Introduction (and similar reasoning works also for the results given in the following). Assume that the function $H_{l}(x)$ (resp. $H_{r}(x)$ ) is given by a composite function $H_{l}(x)=F_{\rho_{p_{l}}} \circ \ldots \circ F_{\rho_{1}}(x)$ (resp. $\left.H_{r}(x)=F_{\sigma_{p_{r}}} \circ \ldots \circ F_{\sigma_{1}}(x)\right)$ where $\rho=\rho_{1} \ldots \rho_{p_{l}}$ (resp. $\left.\sigma=\sigma_{1} \ldots \sigma_{p_{r}}\right)$ is the symbolic sequence associated with a cycle of $F$ of prime period $p_{l}$ (resp. $p_{r}$ ), both colliding with $x=0$ when the parameters are in $P$. Then the proof of Theorem 1 shows that the equations $f_{L}^{k} \circ f_{R}(0)=0$ and $f_{L}^{k} \circ f_{R}\left(x_{0}\right)=x_{0}$,

\footnotetext{
3 and thus constant pieces are excluded
} 
$x_{0}>0$, have a solution for suitable values of $\mu_{l}>0$ and $\mu_{r}<0$ so that, for suitable values of the parameters of the map $F$ (corresponding to the region $\mu_{l}>0$ and $\mu_{r}<0$ as qualitatively shown in Fig. 1), we have that also the solutions of the following equations

$$
\left(F_{\rho_{p_{l}}} \circ \ldots \circ F_{\rho_{1}}\right)^{k} \circ F_{\sigma_{p_{r}}} \circ \ldots \circ F_{\sigma_{1}}\left(x_{0}\right)=x_{0}, x_{0} \geq 0
$$

exist for any $k>0$.

\subsection{Particular cases}

It is clear that the above existence conditions do not regard the stability of the cycles, which depend on the eigenvalues $\lambda\left(R L^{k}\right)$ of the cycles, and thus on the derivatives of the functions $f_{L}$ and $f_{R}$ in the periodic points. However, under specific assumptions we can establish some more results.

Theorem 2. Consider the map $f$ in (2) assuming $f_{L}(x)$ increasing and $f_{R}(x)$ decreasing in $I=\left[f_{R}\left(\mu_{l}\right), \mu_{l}\right]$, for $\mu_{l}>0$ fixed and $\mu_{r}<f_{L} \circ f_{R}\left(\mu_{l}\right)<0$. Then map $f$ can have at most two coexisting stable cycles, with symbolic sequence $R L^{k}$ and $R L^{k+1}$.

Proof. Consider $\mu_{l}>0$ fixed and $\mu_{r} \leq 0$, then the map $f$ is invertible in $I$ as long as $f_{L}\left(f_{R}\left(\mu_{l}\right)\right)>\mu_{r}$, and when this condition is satisfied all the existing cycles of $f$ must be stable. Now let $d_{k}$ be the first preimage of the origin belonging to the positive side, given by $f_{R}^{-1} \circ f_{L}^{-k}(0)=d_{k}$, and thus the fixed point of the first return map $F_{R}(x)$ defined as $T_{0}(x)=f_{L}^{k} \circ f_{R}(x)$ for $x \in\left[0, d_{k}\right]$ exists and is stable, so that a stable cycle $R L^{k}$ for $f$ exists. We know that $T_{0}(0)=f_{L}^{k}\left(\mu_{r}\right) \in J$ and $T_{0}\left(d_{k}\right)=f_{L}^{k} \circ f_{R}\left(d_{k}\right)=0$. Then either $d_{k} \geq \mu_{l}$, in which case the stable cycle $R L^{k}$ is globally attracting in $I$, or $d_{k}<\mu_{l}$ in which case another cycle necessarily exists, with symbolic sequence $R L^{k+1}$, associated with a fixed point of the first return map $T_{1}(x)=f_{L}^{k+1} \circ f_{R}(x)$, for $d_{k}<x \leq \mu_{l}$. That is, necessarily it must be $d_{k+1}>\mu_{l}$ because we know that $T_{1}\left(d_{k}\right)=\mu_{l}, T_{1}\left(d_{k+1}\right)=0$ and the length of $T_{1}^{-1}\left(\left[0, \mu_{l}\right]\right)$ (equal to $\left(d_{k+1}-d_{k}\right)$ ) is larger than the length of $\left[0, \mu_{l}\right]$ (equal to $\left.\mu_{l}\right)$ ).

Also, a sufficient condition to have all stable cycles is that, on the one hand, there exists $\varepsilon_{l}>0$ such that

$$
H_{l}(x) \text { is increasing, } 0 \leq H_{l}^{\prime}(x)<1 \quad \forall x \in\left(-\varepsilon_{l}, 0\right)
$$

and, on the other hand, there exists $\varepsilon_{r}>0$ such that

$$
H_{r}(x) \text { is decreasing, }-1<\left(H_{l} \circ H_{r}\right)^{\prime}(x) \leq 0 \quad \forall x \in\left(0, \varepsilon_{r}\right)
$$

Then, for $\mu_{l}$ and $\mu_{r}$ such that $\left[f_{R}\left(\mu_{l}\right), \mu_{l}\right] \subset\left(-\varepsilon_{l}, \varepsilon_{r}\right)$, the cycles, which exist according to Theorem 1 , must have the eigenvalues $-1<\lambda\left(R L^{k}\right) \leq 0$ for any $k \geq 1$. In such cases, for fixed $\mu_{l}>0$, when we start to decrease the offset $\mu_{r}$ from zero, the first return map has at first no discontinuity point $d_{1}$ in $J$, and a fixed point of $F_{R}$ must exist in $J$ (corresponding to a stable 2-cycle of $f$ ). As $\mu_{r}$ decreases a first bifurcation occurs when $d_{1}=\mu_{l}$ after which $d_{1}<\mu_{l}$ and the first return map is defined by two pieces $T_{0}(x)$ and $T_{1}(x)$ with $k=1$, corresponding to the coexistence of two stable cycles. As $\mu_{r}$ is further decreased, one discontinuity point exit from $J$ leaving one only stable cycle, then another discontinuity point enters in $J$ with $k$ increased by one, leading to bistability and so on. This leads us to conclude that all the stable cycles must necessarily exist, and at most two of them coexist, because at most one discontinuity point $d_{k}$ belongs to $J$, as proved in the following

Theorem 3. Consider the map $f$ in (2) fulfilling the conditions in (9) and (10). Then any neighborhood of the origin $(0,0)$ in the parameter space contains infinitely many periodicity regions $\Pi\left(R L^{k}\right), k \geq 1$, which are issuing from the origin and ordered in anticlockwise direction in the quadrant $\mu_{l}>0$ and $\mu_{r}<0$. In addition, for any $k \geq 1$ every region $\Pi\left(R L^{k}\right)$ has a central part where $R L^{k}$ is the unique attractor and a part where $R L^{k}$ coexists with $R L^{k+1}$.

Proof. From the assumption, let $0<\mu_{l}<\varepsilon_{r}$, then we have that for $\mu_{r}=0, f_{L}\left(f_{R}\left(\left[0, \mu_{l}\right]\right)\right)=f_{L}\left(\left[f_{R}\left(\mu_{l}\right), 0\right]\right)=$ $\left[f_{L}\left(f_{R}\left(\mu_{l}\right)\right), \mu_{l}\right]$ is an interval included in $x>0$, so that the range of $f_{R}$, given by the interval $f_{R}\left(\left[0, \mu_{l}\right]\right)=$ $\left[f_{R}\left(\mu_{l}\right), 0\right]=\left[H_{r}\left(\mu_{l}\right), 0\right]$ has a length smaller than the length of the interval $\left[f_{L}^{-1}(0), 0\right]$. As $f_{L}^{-1}$ is expanding, 

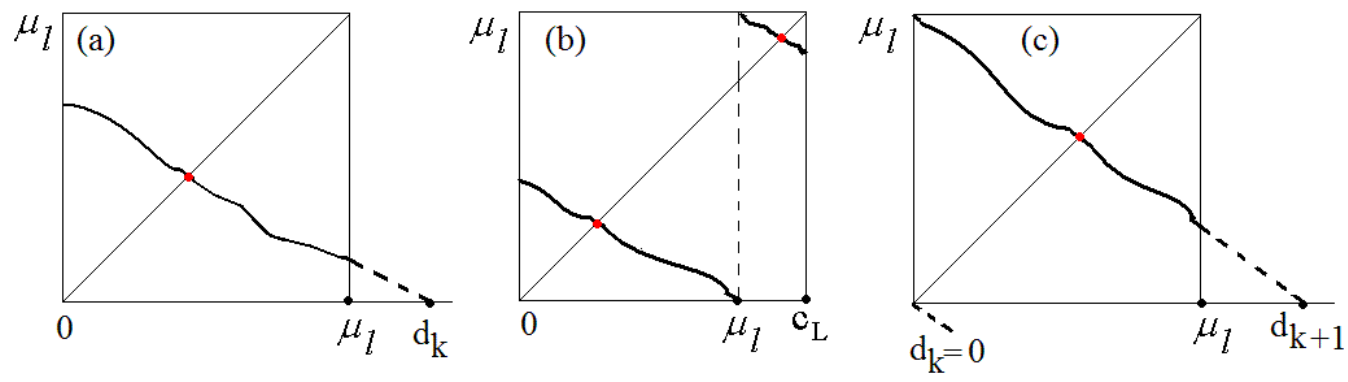

FIgURE 3. First return map $F_{R}$ under the assumptions of Theorem 3.

we have that the length $\mu\left(\left[f_{L}^{-(k+1)}(0), f_{L}^{-k}(0)\right]\right)$ increases with $k$, while for decreasing $\mu_{r}$ the range of the function on the right side is of constant length, as for any $\mu_{r}<0$ we have $f_{R}\left(\left[0, \mu_{l}\right]\right)=\left[\mu_{r}+H_{r}\left(\mu_{l}\right), \mu_{r}\right]$ and $\mu\left(\left[\mu_{r}+H_{r}\left(\mu_{l}\right), \mu_{r}\right]\right)=\mu\left(\left[H_{r}\left(\mu_{l}\right), 0\right]\right)$. This implies that for any $\mu_{r}<0$ the range $f_{R}\left(\left[0, \mu_{l}\right]\right)$ either does not include any preimage of the origin, or it includes at most one preimage. Stated in other words, in the interval $J$ either there is no discontinuity point $d_{k}$ (which means that a unique stable cycle $R L^{k}$ exists) or there is at most one discontinuity point $d_{k} \in J$ (which means that a pair of stable cycles $R L^{k}$ and $R L^{k+1}$ coexist). Notice that when $\mu_{l}>0$ is fixed and small enough, as $\mu_{r}$ is decreased from 0 , then the cycles (which exist for any $k \geq 1$ according to Theorem 1) all appear for increasing values of $k$, thus leading to periodicity regions ordered in anticlockwise direction, and necessarily of stable cycles as $-1<\lambda\left(R L^{k}\right) \leq 0$ for any $k \geq 1$, coexisting in pair in suitable regions.

Note that the proved coexistence between cycles of periods $R L^{k}$ and $R L^{k+1}$ as well as between the cycles $R L^{k+1}$ and $R L^{k+2}$ implies that the existence region of the cycle $R L^{k+1}$ has a central part in which it is the unique attractor, surrounded by two regions in which it coexists (on one side with $R L^{k}$ and on the other side with $\left.R L^{k+2}\right)$.

We remark that this results extends the one presented in [4] as the condition based on the contraction of the right branch is replaced here by the contraction of the composite function $H_{l} \circ H_{r}(x)$ in a right neighborhood of the origin.

The structure of the first return map under the assumptions of Theorem 3 is illustrated in Fig. 3. The BCB occurs when $d_{k}=\mu_{l}$, which means $f_{L}^{k} \circ f_{R}\left(\mu_{l}\right)=0$ that is $f_{L}^{k} \circ f_{R} \circ f_{L}(0)=0$ corresponding to the condition defined in (7). While a stable cycle disappears when $d_{k}=0$, which means $f_{L}^{k} \circ f_{R}(0)=0$ corresponding to the condition defined in (6).

Clearly if the functions $f_{R}(x)$ and $f_{L}(x)$ have a simple structure, then it is also possible to get the BCB curves in analytic form explicitly (from the Eqs. (6) and (7) given in implicit form). For example, considering the piecewise linear case

$$
x^{\prime}=f_{1}(x), \quad f_{1}(x)=\left\{\begin{array}{lll}
f_{L}(x)=\mu_{l}+a_{l} x & \text { if } & x<0 \\
f_{R}(x)=\mu_{r}+a_{r} x & \text { if } & x>0
\end{array}\right.
$$

it is very easy to get the BCB curves explicitly in analytic form (the results are shown in Fig. 4a).

One more example is given by the following piecewise smooth map

$$
x^{\prime}=f_{2}(x), f_{2}(x)=\left\{\begin{array}{lll}
f_{L}(x)=\mu_{l}+a\left(e^{x}-1\right) & \text { if } \quad x<0 \\
f_{R}(x)=\mu_{r}+b\left(e^{-x}-1\right) & \text { if } \quad x>0
\end{array}\right.
$$

which fulfills the conditions of Theorem 3 for $0<a b<1$. For the case $a=0.7$ and $b=1.3$ the periodicity regions of this map are shown in Fig. 4b.

As already remarked, in general the stability of the maximal cycles depends on the derivatives in the periodic points. However, as long as the first return map is uniquely invertible in $I$, unstable cycles (and therefore chaos) 

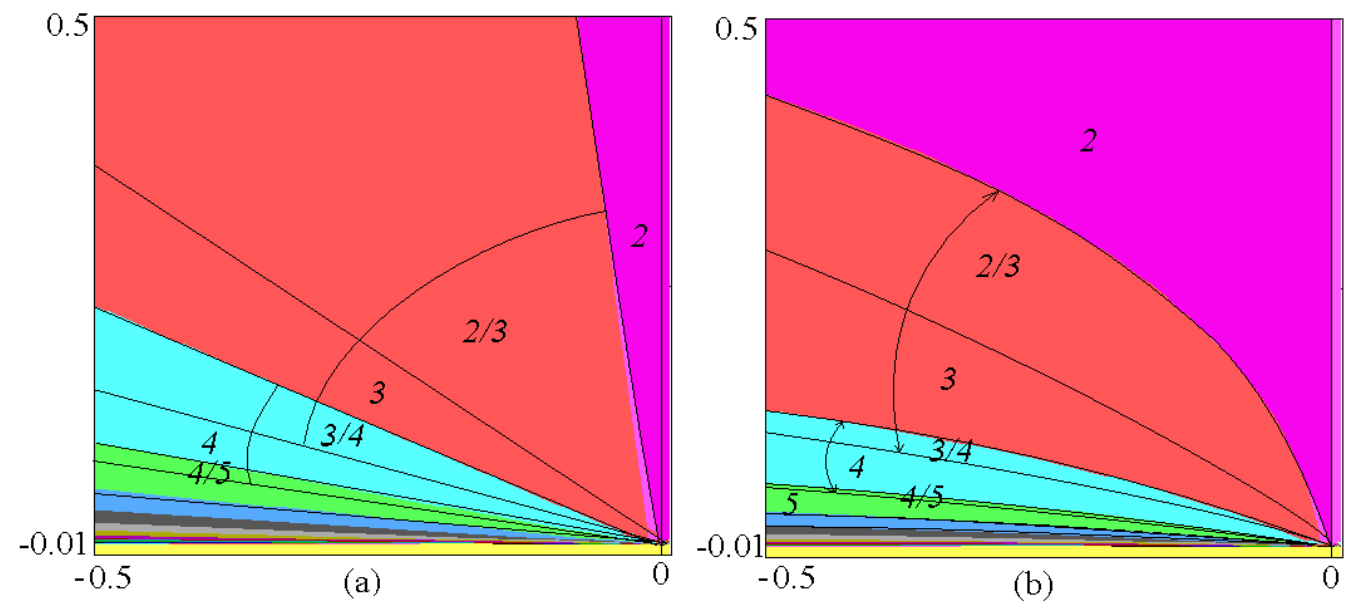

FiguRE 4. Bifurcation scenario in the $\left(\mu_{r}, \mu_{l}\right)$ parameters space. (a) System (11) with $a_{l}=0.7$ and $a_{r}=-1.3$. (b) System (12) with $a=0.7$ and $b=1.3$.

cannot occur. As stated by Theorem 2 only stable cycles can exist, at most pairwise coexisting. While when the first return map is non uniquely invertible in $I$ then chaos can occur. As an example consider the map

$$
x^{\prime}=f_{3}(x), \quad f_{3}(x)= \begin{cases}f_{L}(x)=\mu_{l}+a x & \text { if } \quad x<0 \\ f_{R}(x)=\mu_{r}+b\left(e^{-x}-1\right) & \text { if } \quad x>0\end{cases}
$$

whose graph for $a=1.2$ and $b=1.5$ is shown in Fig. 5. In this case we can have the analytic BCB curves of the maximal cycles. In fact we have

and

$$
f_{L}^{k}(x)=\mu_{l} \frac{1-a^{k}}{1-a}+a^{k} x
$$

$$
f_{L}^{k} \circ f_{R}(x)=\mu_{l} \frac{1-a^{k}}{1-a}+a^{k} \mu_{r}+b a^{k}\left(e^{-x}-1\right)
$$

so that the BCB curves defined by the condition $f_{L}^{k} \circ f_{R}(0)=0$ are given by

and from

$$
\xi_{k}^{r}: \mu_{l}=-\mu_{r}\left(a^{k} \frac{1-a}{1-a^{k}}\right)
$$

$$
f_{L}^{k-1} \circ f_{R} \circ f_{L}(x)=\mu_{l} \frac{1-a^{k-1}}{1-a}+a^{k-1} \mu_{r}+b a^{k-1}\left(e^{-\left(\mu_{l}+a x\right)}-1\right)
$$

we obtain the BCB curves defined by the condition $f_{L}^{k-1} \circ f_{R} \circ f_{L}(0)=0$ which are given by

$$
\left.\xi_{k}^{l}: \mu_{r}=-b\left(e^{-\mu_{l}}-1\right)-\mu_{l} \frac{1-a^{k-1}}{(1-a) a^{k-1}}\right)
$$

shown in Fig. 6 for $k=1, \ldots, 5$.

Let us close this section showing another example in which the function $H_{l}(x)=(x+1)^{2}-1$ leads to an unstable fixed point $x_{l}^{*}\left(\mu_{l}\right)$ for $f_{L}(x)$. Consider

$$
x^{\prime}=f_{4}(x), f_{4}(x)=\left\{\begin{array}{ll}
f_{L}(x)=\mu_{l}+(x+1)^{2}-1 & \text { if } \quad x<0 \\
f_{R}(x)=\mu_{r}-0.1 x & \text { if } \quad x>0
\end{array} .\right.
$$



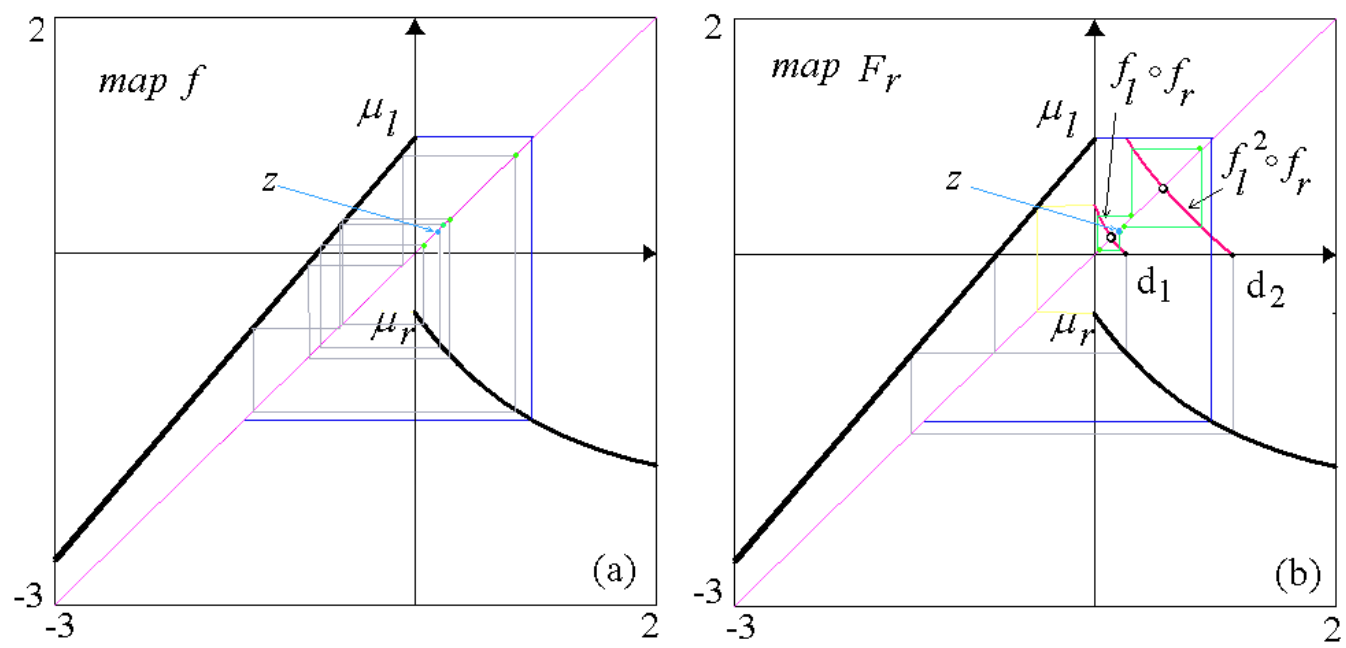

Figure 5. In (a) graph of the map $f_{3}$ in (13) and of the first return map in (b), at $a=1.2$, $b=1.5, \mu_{l}=1$ and $\mu_{r}=-0.5$.
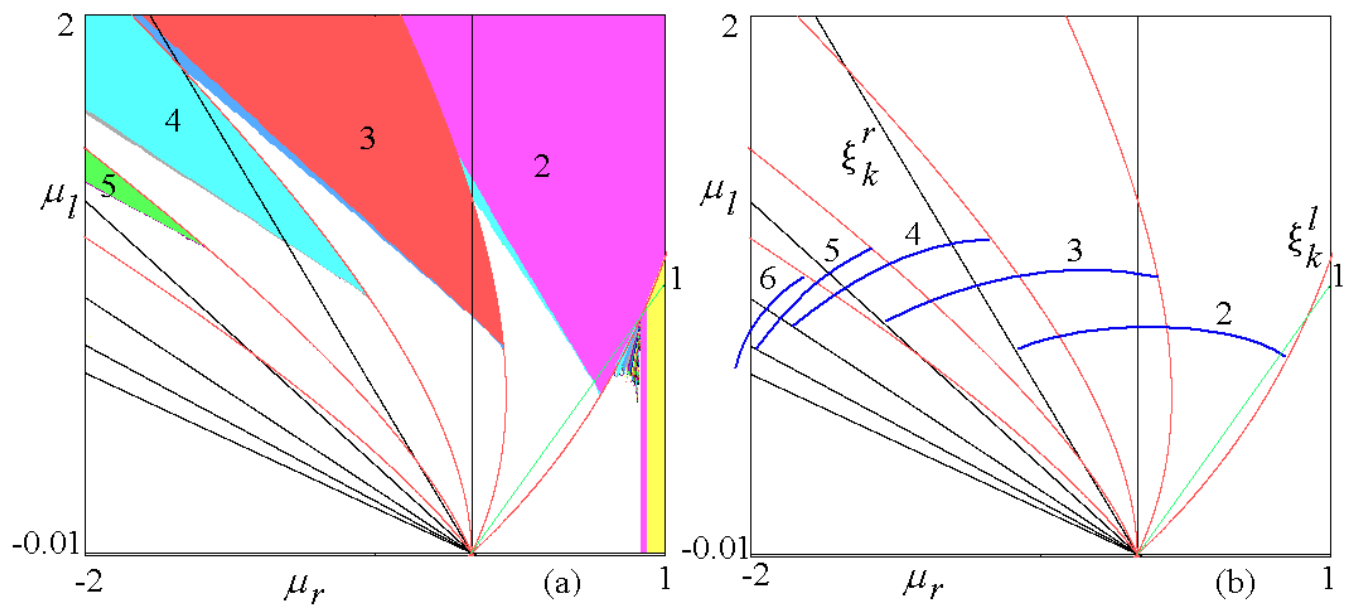

Figure 6. Bifurcation scenario for example (13) in the $\left(\mu_{r}, \mu_{l}\right)$ parameter space for $a=1.2$ and $b=1.5$. In (a) periodicity regions of stable cycles, which are overlapping in pair for $a b>1$. The white region corresponding to chaotic behavior. In (b) the BCB curves whose analytical expressions are given in (18) and (16).

In Fig. 7a we show a two-dimensional bifurcation diagram of this map, which in large is dominated by the quadratic function $H_{l}(x)$. However, locally (in a neighborhood of $(0,0)$ in the parameter plane) we have $H_{l}(x)$ increasing, so that Theorem 1 can be applied, and we shall see the BCB curves issuing from $(0,0)$ which are accumulating on the constraint in (4). Here we have $f_{R} \circ f_{L}(0)=-0.1 \mu_{l}+\mu_{r}$, the unstable fixed point is $x_{l}^{*}\left(\mu_{l}\right)=\frac{-1+\sqrt{1-4 \mu_{l}}}{2}$ so that the periodicity regions issuing from $(0,0)$ in the parameter plane belong to the region satisfying

$$
-0.1 \mu_{l}+\mu_{r}>\frac{-1+\sqrt{1-4 \mu_{l}}}{2}
$$




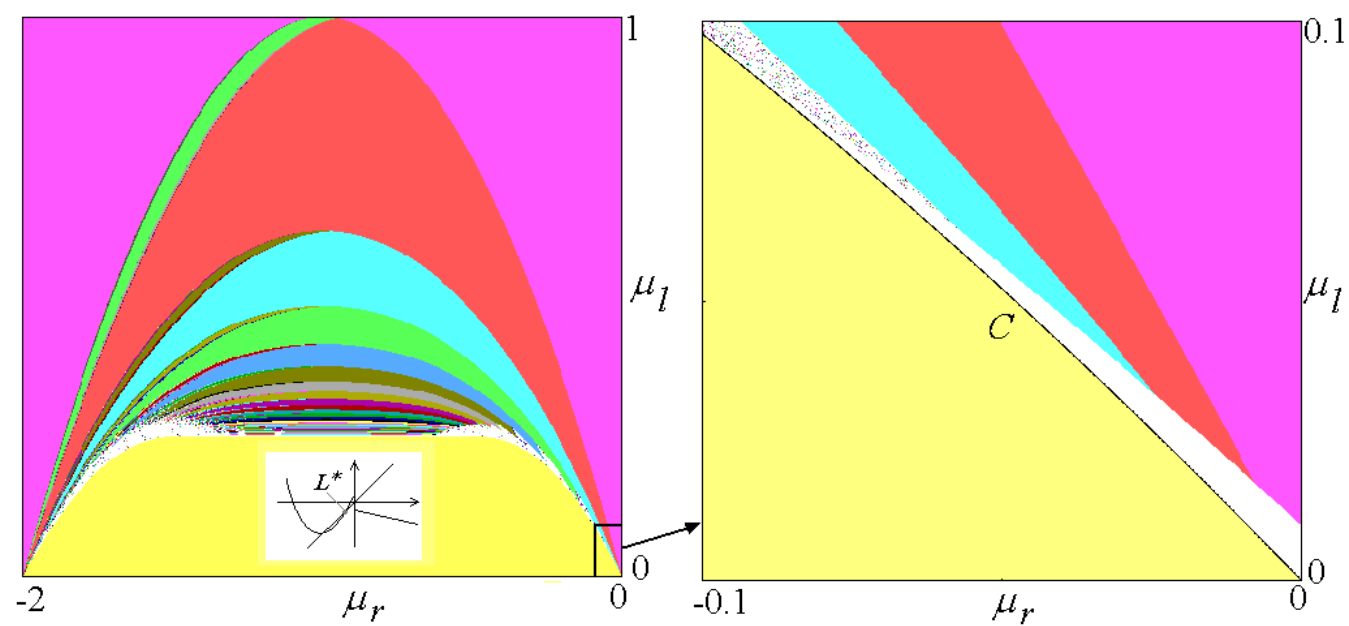

FiguRE 7. Bifurcation scenario for example (19) in the $\left(\mu_{r}, \mu_{l}\right)$ parameter space.

and only a few of them are associated with stable cycles. In any case the curves are accumulating on the set denoted as $C$ in Fig. $7 \mathrm{~b}$, given by the equation

$$
C: \quad \mu_{r}=0.1 \mu_{l}+\frac{-1+\sqrt{1-4 \mu_{l}}}{2}
$$

and the white region shown in Fig. 7b is associated with a chaotic behavior.

\subsection{Particular case $f_{R}(x)=$ const}

This particular case is quite simple. The periodicity regions associated with $k$-cycles described above all exist without overlapping. In fact, considering

$$
f_{R}(x)=\mu_{r} \text { for any } x \geq 0
$$

and $f_{L}(x)$ increasing in $\left[\mu_{r}, 0\right]$, we have that the range of $f_{R}(x)$ is the unique point $\mu_{r}$ and the first return map is given by a constant function with the value $f_{L}^{k}\left(\mu_{r}\right)>0$ for a suitable $k \geq 1$, which is then mapped into a periodic point (as we necessarily have $f_{R} \circ f_{L}^{k}\left(\mu_{r}\right)=\mu_{r}$ ). It is clear that fixed any value $\mu_{l}>0$, then decreasing $\mu_{r}$ all the $n$-cycles, for any integer $n \geq 2$ can be obtained. In fact, given $\mu_{l}$, consider the value $f_{L}^{-1}(0)$, then for $f_{L}^{-1}(0) \leq \mu_{r}<0$ we have a $2-$ cycle, for $f_{L}^{-2}(0) \leq \mu_{r}<f_{L}^{-1}(0)$ we have a 3 -cycle, and so on, defining $f_{L}^{-0}(0)=0$, for $f_{L}^{-k}(0) \leq \mu_{r}<f_{L}^{-(k-1)}(0), k \geq 1$, we have a $(k+1)$-cycle with symbolic sequence $R L^{k}$. The $\mathrm{BCB}$ curves of the cycles are given by

$$
\mu_{r}=f_{L}^{-k}(0)
$$

\section{The QUADRANT $\mu_{l}>0$ AND $0<\mu_{r}<\mu_{l}$}

Let us here assume that $H_{l}(x)$ fulfills the conditions stated in Eq. (9) and that $H_{r}(x)$ is a decreasing function in a right neighborhood of $x=0$. As we have seen in Sec. 2, at $\mu_{r}=0$ the BCB of the fixed point $x_{r}^{*}$ occurs and a 2-cycle of $f$ exists (at least for $\mu_{l}$ close to 0 ). Thus the BCB curve leading to the appearance of the 2-cycle belongs to the region with $\mu_{l}>0$; and a region of coexistence between the fixed point $x_{r}^{*}$ and the 2-cycle must exist. Only in the case $f_{R}(x)=\mu_{r}$ (constant function) the BCB curve corresponding to the creation of the cycle $R L$ is the same as the destruction of the fixed point $x_{r}^{*}$ and it is the line $\mu_{r}=0$. Otherwise, this BCB curve 

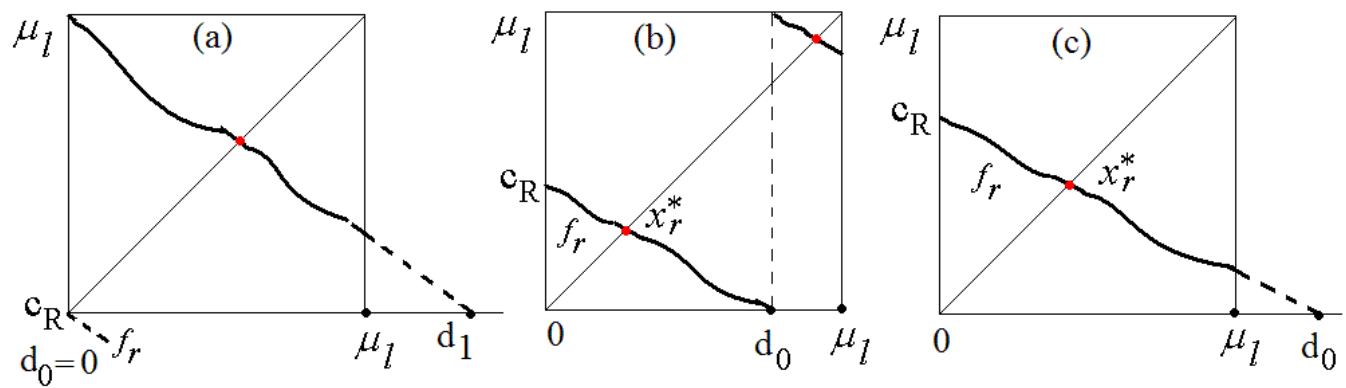

FiguRE 8. Different situations for the first return map $F_{R}$ when $x_{r}^{*}$ is stable.
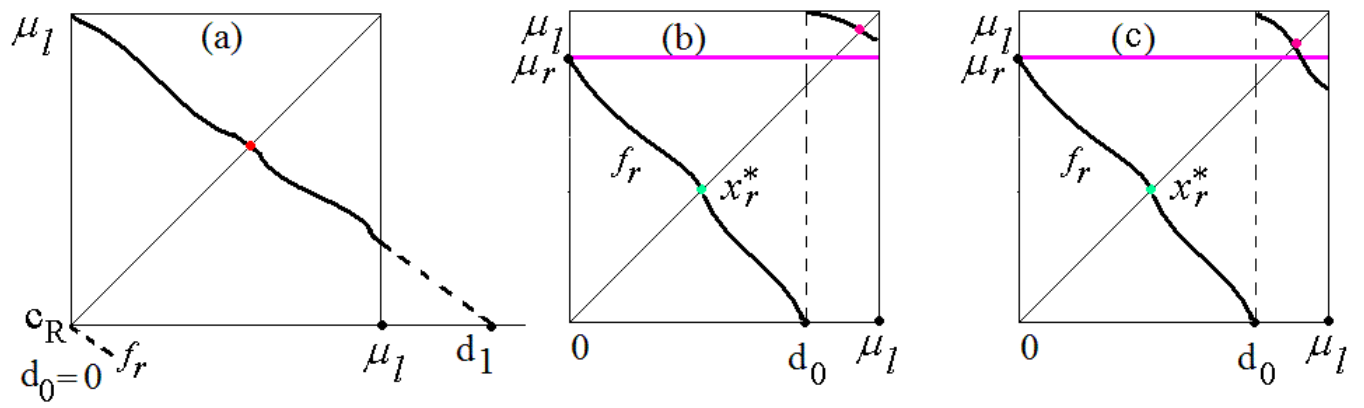

Figure 9. Different situations for the first return map $F_{R}$ when $x_{r}^{*}$ is unstable.

has a portion in the quadrant $\mu_{r}>0$. Here the dynamical behaviors are different and depend on the existence of a stable or unstable fixed point $x_{r}^{*}$ on the right side. In any case we can investigate the dynamics by using again the first return map.

For $\mu_{r}=0$ we have $f_{r}(0)=0$ (see Fig. 8a) and thus for $\mu_{r}>0$ a fixed point $x_{r}^{*}$ exists in $x>0$ which may be locally stable or unstable. Let us distinguish between these two cases

(I) $x_{r}^{*}$ is stable. For $\mu_{r}=0$ we have $d_{1}=f_{R}^{-1} \circ f_{L}^{-1}(0)>\mu_{l}$ (as $f_{L}^{-1}$ is expanding) and the point $d_{0}=f_{R}^{-1}(0)=0$, so that the first return map in the interval $J=\left[0, \mu_{l}\right]$ is given by the decreasing function $f_{L} \circ f_{R}(x)$ which has a stable fixed point (a 2-cycle for the original map). For $\mu_{r}>0$ we have

$$
d_{0}=f_{R}^{-1}(0)>0
$$

and clearly $d_{0}$ is a discontinuity point. For $x \in\left(0, d_{0}\right)$ the map is positive, decreasing and a stable fixed point $x_{r}^{*}$ exists whose basin in the positive side is $\left(0, d_{0}\right)$, so that here the first return map is the map itself, while the first return map in the interval $\left(d_{0}, \mu_{l}\right]$ is given by $f_{L} \circ f_{R}(x)$ and has a stable fixed point (a 2-cycle for the map $f)$ as $f_{L} \circ f_{R}\left(d_{0}\right)=\mu_{l}$ and $f_{L} \circ f_{R}\left(\mu_{l}\right)>0$ (see Fig. 8b). Thus we have the coexistence of two attracting cycles, the fixed point $x_{r}^{*}$ and a 2-cycle with symbolic sequence $R L$. Increasing $\mu_{r}>0$ also $d_{0}$ increases and the BCB leading to the disappearance of the 2 -cycle occurs when $d_{0}=\mu_{l}$, i.e. when $f_{R}^{-1}(0)=\mu_{l}$, which we can write also as

$$
f_{R}\left(\mu_{l}\right)=0 \quad \text { or } \quad f_{R} \circ f_{L}(0)=0
$$

and after that, for higher values of $\mu_{r}$ we have a unique stable fixed point $x_{r}^{*}$.

(II) $x_{r}^{*}$ is unstable. For $\mu_{r}=0$ we have $d_{1}=f_{R}^{-1} \circ f_{L}^{-1}(0)>\mu_{l}$ and the point $d_{0}=f_{R}^{-1}(0)=0$, so that the first return map in the interval $\left[0, \mu_{l}\right]$ is given by the decreasing function $f_{L} \circ f_{R}(x)$ which has a stable fixed 

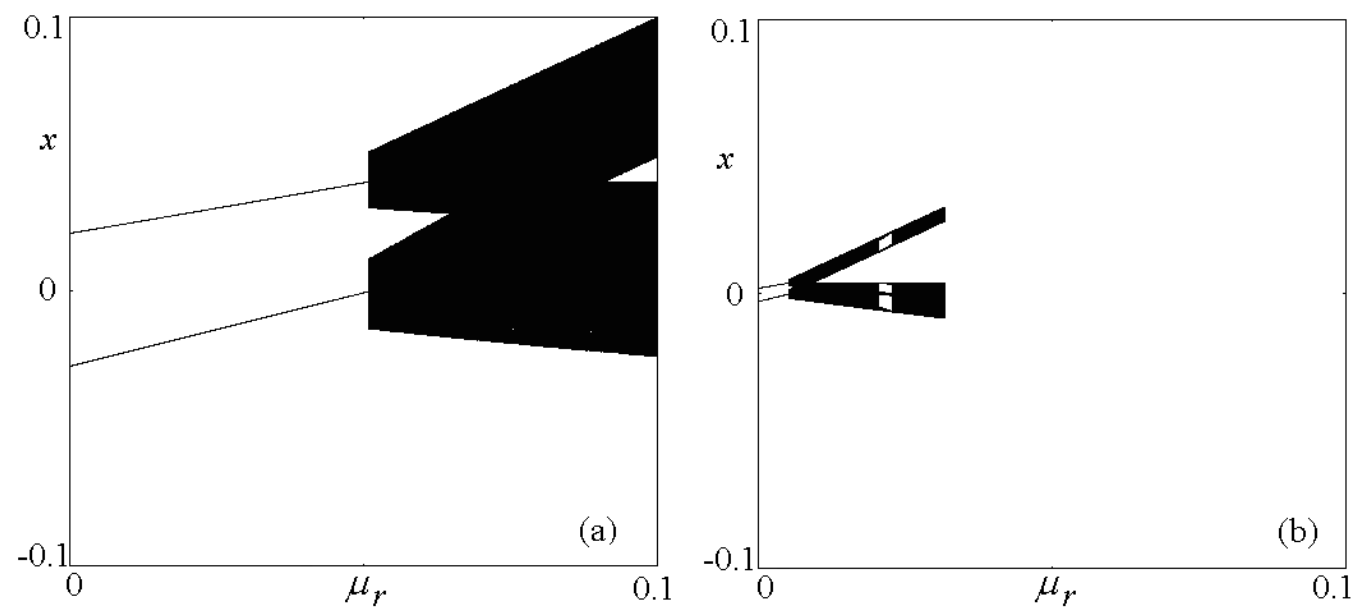

FiguRE 10. One-dimensional bifurcation diagrams for example (12) with $a=0.7$ and $b=1.3$. (a) $\mu_{l}=0.04$ and (b) $\mu_{l}=0.004$.
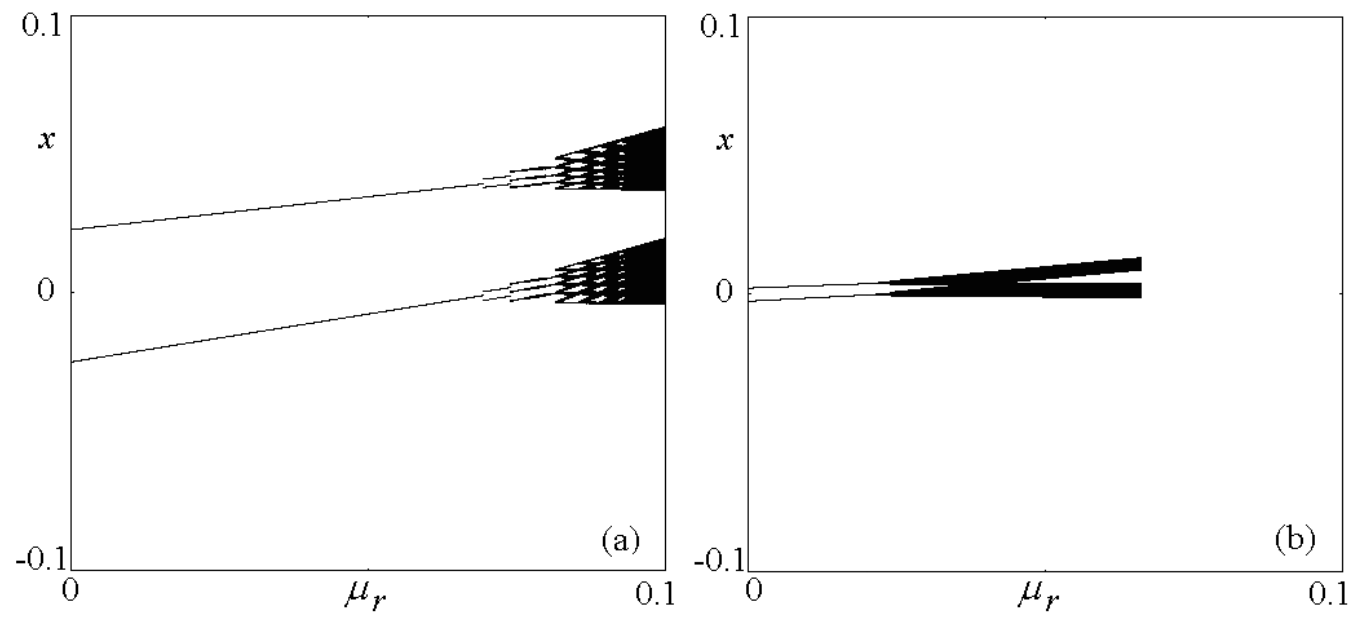

FiguRE 11. One-dimensional bifurcation diagrams for example (12) with $a=0.7$ and $b=1.1$. (a) $\mu_{l}=0.04$ and (b) $\mu_{l}=0.004$.

point (a 2-cycle for the original map) (see Fig. 9a). For $\mu_{r}>0$ we have a discontinuity point in

$$
d_{0}=f_{R}^{-1}(0)>0 .
$$

For $x \in\left(0, d_{0}\right)$ the map is positive, decreasing and an unstable fixed point $x_{r}^{*}$ exists. As long as $d_{0}<\mu_{l}$ for $x \in\left(0, d_{0}\right)$ the first return map is the map itself, while the first return map in the interval $\left(d_{0}, \mu_{l}\right]$ is given by $f_{L} \circ f_{R}(x)$ and has a stable fixed point (a 2-cycle for the map $f$ ). Thus the behavior of the map $f$ for points $x \in\left(0, d_{0}\right)$ depends on the shape of $f_{R}$. However, we recall that a decreasing function besides the fixed point $x_{r}^{*}$, can have at most cycles of period 2 in pair, alternated stable and unstable. Also if $f_{L} \circ f_{R}\left(\mu_{l}\right)>\mu_{r}$ then the first return map is invertible (Fig. 9b), which means that chaos cannot occur, while for $f_{L} \circ f_{R}\left(\mu_{l}\right)<\mu_{r}$ (Fig. 9c) chaos can occur. Clearly the BCB leading to the disappearance of the 2-cycle occurs, as before, at $d_{0}=\mu_{l}$, i.e. as in Eq. (23). 
Examples of one-dimensional bifurcation diagrams with the exponential functions in (12) are given in Figs. 10 and 11 .

\section{REFERENCES}

[1] V. Avrutin, M. Schanz, On multi-parametric bifurcations in a scalar piecewise-linear map, Nonlinearity 19 (2006) 531-552.

[2] V. Avrutin, M. Schanz, S. Banerjee, Multi-parametric bifurcations in a piecewise-linear discontinuous map, Nonlinearity 19 (2006) 1875-1906.

[3] V. Avrutin, M. Schanz, S. Banerjee, Codimension-3 bifurcations: Explanation of the complex 1-, 2- and 3d bifurcation structures in nonsmooth maps, Physical Review E 75 (2007) 066205.

[4] Avrutin V, A. Granados, and M. Schanz, Sufficient conditions for a period increment big bang bifurcation in one-dimensional maps. Nonlinearity 24(9) 2575-2598, 2011.

[5] V. Avrutin, M. Schanz, L. Gardini, Computation of bifurcation curves by map replacement, International Journal of Bifurcation and Chaos, 20(10) 3105-3135, 2010.

[6] S. Banerjee, M. S. Karthik, G. Yuan, and J.A. Yorke. Bifurcations in one-dimensional piecewise smooth maps-theory and applications in switching circuits. IEEE Trans. Circ. \& Sys. I, 47:389-394, 2000.

[7] G.I. Bischi, L. Gardini, U. Merlone "Impulsivity in binary choices and the emergence of periodicity", Discrete Dynamics in Nature and Society, Article ID 407913, 22 pages, doi:10.1155/2009/407913 (2009).

[8] M. di Bernardo, M.I. Feigin, S.J. Hogan, M.E. Homer, Local analysis of C-bifurcations in ndimensional piecewise smooth dynamical systems, Chaos, Solitons \& Fractals 10 (1999) 1881-1908.

[9] M. di Bernardo, C.J. Budd, A.R. Champneys, P. Kowalczyk, Piecewise-smooth Dynamical Systems: Theory and Applications, Applied Mathematical Sciences 163, Springer-Verlag, London, 2007.

[10] L. Gardini, F. Tramontana, V. Avrutin, M. Schanz, Border Collision Bifurcations in 1D PWL map and the Leonov approach, International Journal of Bifurcation and Chaos, 20(10) 3085-3104, 2010.

[11] N. N. Leonov, Map of the line onto itself, Radiofisica 3 (1959) 942-956.

[12] N. N. Leonov, "On a discontinuous piecewise-linear pointwise mapping of a line into itself" Radiofisika, 3(3), 496-510, 1960.

[13] C. Mira, Chaotic dynamics, World Scientific, Singapore, 1987.

[14] Y.L. Maistrenko, V.L. Maistrenko, L.O. Chua, Cycles of chaotic intervals in a time-delayed Chua's circuit, International Journal of Bifurcation and Chaos 3 (1993) 1557-1572.

[15] Y.L. Maistrenko, V.L. Maistrenko, S.I. Vikul, L.O. Chua, Bifurcations of attracting cycles from time-delayed Chua's circuit, International Journal of Bifurcation and Chaos 5 (1995) 653-671.

[16] Y.L. Maistrenko, V.L. Maistrenko, S.I. Vikul, On period-adding sequences of attracting cycles in piecewise linear maps, Chaos, Solitons \& Fractals 9 (1998) 67-75.

[17] H.E. Nusse, J.A. Yorke, Border-collision bifurcations including period two to period three for piecewise smooth systems, Physica D 57 (1992) 39-57.

[18] H.E. Nusse, J.A Yorke, Border-collision bifurcation for piecewise smooth one-dimensional maps, International Journal of Bifurcation and Chaos 5 (1995) 189-207.

[19] F.Tramontana, F.Westerhoff, L.Gardini "On the complicated price dynamics of a simple one-dimensional discontinuous financial market model with heterogeneous interacting traders", Journal of Economic Behavior and Organization , 74 (2010) $187-205$

[20] F. Tramontana, L. Gardini, A. Agliari "Endogenous cycles in discontinuous growth models" Mathematics and Computers in Simulation, , 81 (2011) 1625-1639.

[21] F. Tramontana, L. Gardini, T. Puu "Global Bifurcations in a Piecewise-Smooth Cournot Duopoly Game", Chaos, Solitons \& Fractals 43 (2010) 15-24

[22] F. Tramontana, L. Gardini, V. Avrutin, M. Schanz "New Adding Phenomena in Piecewise Linear Maps with two discontinuities." International Journal of Bifurcation and Chaos (To appear) 2011.

[23] Zhusubaliyev, Z. T. \& Mosekilde, E. [2003] Bifurcations and Chaos in Piecewise-Smooth Dynamical Systems (World Scientific, Singapore). 